\title{
Functional Mapping of Horizontal Connections in Developing Ferret Visual Cortex: Experiments and Modeling
}

\author{
Michael Weliky and Lawrence C. Katz \\ Department of Neurobiology, Duke University Medical Center, Durham, North Carolina 27710
}

In cat striate cortex, patchy horizontal axonal projections link columns of similar orientation specificity. To assess the physiological correlates of such clustered projections, a new multisite stimulation technique was used to functionally map the pattern of horizontal synaptic inputs onto single layer $2 / 3$ cells within tangential slices of developing ferret visual cortex. Twenty-four separate sets of horizontal fibers were stimulated within a $1200 \mu \mathrm{m}$ strip of cortex, while evoked synaptic responses were recorded using whole-cell patch methods. For most cells, input maps demonstrated the presence of clustered horizontal connections in which multiple strong and weak synaptic responses were alternately evoked across the stimulated cortical region. Recordings from up to nine cells in a single slice revealed that patterns of synaptic input were closely correlated for cells in close proximity, and that this correlation decreased with distance, with no correlation at distances greater than $500 \mu \mathrm{m}$.

To determine whether these physiological results were consistent with the known anatomical linkage of iso-orientation columns by clustered horizontal connections, mathematical analysis and computer simulations were performed upon orientation tuning maps obtained from optical imaging of activity-dependent intrinsic signals in mature ferret visual cortex. Optical imaging revealed an organization of iso-orientation domains consisting of broad regions of cortex across which orientation preference smoothly varied, together with “orientation centers" around which orientation preference was arranged in a pinwheel manner. The distribution of synaptic connections between different cortical sites was simulated by a model of functionally linked iso- orientation columns. Simulated synaptic input maps, generated by the same stimulation and recording arrangements used in our experimental protocol, accurately reproduced the observed patterns of clustered inputs onto experimentally recorded cells. These results indicate that even at the time of eye opening, prior to extensive visual experience, most cells receive patterns of synaptic inputs consistent with a clustered organization of horizontal connections that functionally link isoorientation columns.

Received Jan. 24, 1994; revised Apr. 25, 1994; accepted June 1, 1994.

We thank Drs. Dale Purves, David Fitzpatrick, Karl Kandler, and Leonard White for critical comments on the manuscript. Also thanks to Drs. David Fitzpatrick and Brett Schofield for help with the optical imaging, and Scott Douglas for technical assistance. This work was supported by EY07960.

Correspondence should be addressed to Michacl Weliky, Department of Ncurobiology, Duke University Medical Center, Box 3209, Durham, NC 27710.

Copyright $(1994$ Society for Neuroscience $0270-6474 / 94 / 147291-15 \$ 05.00 / 0$
[Key words: ferret, horizontal connections, modeling, visual cortex, development, computer simulation, multielectrode stimulation, optical imaging, intrinsic signals]

Throughout the mammalian neocortex, patchy long-range horizontal connections link spatially separate regions within a cortical area (Gilbert and Wiesel, 1979; Rockland and Lund, 1982, 1983; Livingstone and Hubel, 1984; Martin and Whitteridge, 1984). In cat primary visual cortex, clustered connections originate primarily from pyramidal cells (Gilbert and Wiesel, 1979, 1983; Martin and Whitteridge, 1984) and preferentially interconnect columns of similar orientation specificity (Gilbert and Wiesel, 1989; Malach et al., 1993). The adult pattern of clustered connections in visual cortex emerges gradually from an initially unclustered network of horizontal axon collaterals present in the early postnatal cortex. In cats, in which the emergence of clustered connections has been most extensively studied, anatomical studies using retrograde tracers and intracellular dye injections reveal that horizontal connections are unclustered during the first postnatal week, prior to eye opening. Just around the time of eye opening, crude clusters emerge, which are progressively refined and elaborated over the following 6 weeks (Callaway and Katz, 1990; Lubke and Albus, 1992a,b). Activitydependent mechanisms have been shown to organize the pattern of horizontal connections during this refinement period (Callaway and Katz, 1991; Löwel and Singer, 1992).

Although clustered horizontal connections in developing visual cortex have been examined anatomically, the relationship between anatomical projections and spatial patterns of functional synaptic connectivity and plasticity is poorly understood. Cross-correlation methods have demonstrated functional horizontal interactions within developing and mature cat visual cortex (Tso et al., 1986; Hata et al., 1993; however, this approach cannot determine the patterns of these connections with fine spatial resolution. In order to examine the spatial distribution of functional horizontal connections, we have used wholecell patch-clamp recording, in conjunction with a new multisite electrical stimulation technique, to map patterns of long-range horizontal synaptic inputs onto single neurons in ferret visual cortex. We focused on the state of horizontal connections in layer $2 / 3$ of animals around the time of eye opening, postnatal day (P) 28-34, which in the cat corresponds to the time at which crude clusters emerge. These experiments focused on whether the patterns of horizontal synaptic connectivity in these young cortices are consistent with the presence of clustered connections that link iso-orientation columns. To investigate this issue, we experimentally mapped the pattern of synaptic inputs onto single cells using a large multielectrode stimulation array. These 
patterns were then compared to computer simulations of clustered connections predicted by a model of linked iso-orientation columns. Computer simulations were performed upon orientation tuning maps obtained from optical imaging of activitydependent intrinsic signals from adult ferret visual cortex. In the simulations, cortical sites with the same orientation tuning were strongly connected, with the connection strength decreasing between sites having progressively more orthogonal orientation tuning preferences. Our results indicate that even at the time of eye opening, prior to extensive visual experience, most cells receive patterns of synaptic inputs consistent with a clustered organization of horizontal connections that functionally link iso-orientation columns.

\section{Materials and Methods}

Slice preparation. Ferrets (P28-34) were anesthetized with Nembutal $(60 \mathrm{mg} / \mathrm{kg}$, i.p.) and decapitated. The skull and dura were reflected to expose the brain. The brain was removed and placed into ice-cold oxygenated sucrose-artificial cerebral spinal fluid (ACSF) (see below for composition). A block of the mediolateral occipital cortex, containing visual areas 18 and 17 , was cut from the surrounding tissue and the pia gently removed. This tissue block was placed into the vibratome chamber and immediately covered with ice-cold oxygenated sucrose-ACSF. Tangential slices were cut at $350 \mu \mathrm{m}$ thickness, maneuvered onto small shcets of membranc filter paper (Nuclcporc Corp., Plcasanton, CA) (12 $\mu \mathrm{m}$ pore size), and then transferred to an interface chamber held at $33^{\circ} \mathrm{C}$. Only slices obtained from the first two tangential cuts were used, to ensure that only layers 2,3 , and parts of layer 4 would be contained within the slices. Slices were maintained in a heated interface chamber in an atmosphere of $95 \% \mathrm{O}_{2}, 5 \% \mathrm{CO}_{2}$. After $1 \mathrm{hr}$, the sucrose-ACSF was replaced by standard ACSF. After at least another hour, slices were transferred to a recording chamber where they were submerged in a constantly perfused solution of oxygenated ACSF (flow rate $>1 \mathrm{ml}$ / min, recording chamber volume $\sim 1 \mathrm{ml}$ ) at room temperature.

Electrophysiological recording and stimulation. Whole-cell patch electrodes (5-9 M $\Omega$ ) were pulled from capillary glass tubing. Conventional "blind patching" methods and instrumentation were used to obtain gigaohm seals (Blanton et al., 1989). After rupture of the cell membrane, cclls wcre held at $-66 \mathrm{mV}$ and the holding current monitored continuously. Slices were viewed in the recording chamber with a dissecting scope fitted with a gridded reticule in the eyepiece, which allowed the exact location of recording sites to be determined.

The multielectrode stimulation array consisted of 24 monopolar electrodes arranged in a single row with approximately $50 \mu \mathrm{m}$ separation (center to center) between adjacent electrodes. Each stimulating electrode consisted of a $25-\mu \mathrm{m}$-diameter tungsten wire (California Fine Wire, Grover City, CA) insulated except at the tip. The overall length of the array was $1200 \mu \mathrm{m}$. The electrode array was positioned parallel to the top surface of the slice and the electrode tips were inserted approximately $100-200 \mu \mathrm{m}$ into the slice (Fig. 1A). Before each experiment, the resistance of each stimulating electrode was checked by applying single short-duration current pulses to ensure that the resistances of all clectrodes did not vary by morc than $10-20 \%$. It was important that all electrode resistances were approximately equal, ensuring that the tip exposure, and therefore the current passed by each electrode for a given voltage, would be equivalent.

To ensure that distinct cell groups were activated by the stimulation array, we determined the extent to which sets of cells stimulated by adjacent electrodes were separate and nonoverlapping by applying the method of occlusion (Bradler and Barrionuevo, 1989). This was done in four different slices at the same stimulus intensities used in the experiments $(4-9 \mathrm{~V})$. Under voltage clamp, the response evoked by simultancous activation of any two inputs was compared to the algebraic sum of the responses evoked by activation of the inputs separately. If overlapping groups of cells were stimulated, the algebraic sum would be greater than the simultaneously evoked response of the two inputs. We could not detect any significant difference between evoked and summed responses $(t$ test, $P<0.05$ ). This indicates that stimulation electrodes activated separate cell populations. A histogram of the mean differences between evoked and summed responses for electrodes separated by distances up to $200 \mu \mathrm{m}$ (four electrode separation) demonstrates that these differences were negligible (Fig. $1 E$ ).

Electrical stimuli consisted of square pulses of $100 \mu \mathrm{sec}$ duration ranging from 4 to $9 \mathrm{~V}$. Single test pulses were delivered to successive electrodes at a rate of $0.2 \mathrm{~Hz}$. Synaptic responses were recorded in voltage-clamp mode (Axopatch-1D). Stimulus intensities were adjusted so that synaptic responses were below spike-generating thresholds. When more than one cell was recorded from in a single slice, we attempted to maintain the same distance between the stimulation array and each recorded cell.

Solutions. The standard ACSF contained, in $\mathrm{mM}, \mathrm{NaCl}, 125 ; \mathrm{MgSO}_{4}$, 1.3; $\mathrm{CaCl}_{2}, 3.2 ; \mathrm{KCl}, 5 ; \mathrm{KH}_{2} \mathrm{PO}_{4}, 5.2$; dextrose, 10 ; and $\mathrm{NaHCO}_{3}, 25.7$. In sucrose-ACSF, $\mathrm{NaCl}$ was replaced by $248 \mathrm{~mm}$ sucrose (Aghajanian and Rasmussen, 1989). Both solutions were saturated with $95 \% \mathrm{O}_{2}, 5 \%$ $\mathrm{CO}_{2}$. Internal solution for the recording electrodes contained, in $\mathrm{mM}$, D-gluconic acid, 110; $\mathrm{CsOH}, 110 ; \mathrm{EGTA}, 11 ; \mathrm{CsCl}_{2}, 10 ; \mathrm{MgCl}_{2}, 1 ; \mathrm{CaCl}_{2}$, 1; IIEPES, 10; ATP, 0.3; GTP, 1.8 .

Analysis and construction of synaptic input maps. For each input map, 3-10 stimulation trials were averaged from each electrode. To visualize more easily the large-scale structure in these maps, spatial averaging was used to smooth the data acquired from the 24 electrodes and was overlaid upon the original data (Fig. $1 D$ ). This was done by performing a weighted sum of each response with its two nearest neighbors according to the following scheme: each of the two neighbors' responses were weighted by $20 \%$ while the central response was weighted by $60 \%$ before summing (Fig. $1 C, D$ ). This procedure left the overall shape and largescale structure of the pattern intact, but reduced the contribution of small fluctuations in the pattern.

To determine quantitatively the similarity of synaptic input maps, the Pearson product-moment correlation coefficient $r$ was calculated between pairs of maps (Glantz, 1981):

$$
r=\Sigma\left(\left(X-X_{M}\right)\left(Y-Y_{M}\right)\right) /\left(\Sigma\left(X-X_{M}\right)^{2} \Sigma\left(Y-Y_{M}\right)^{2}\right)^{1 / 2}
$$

where $X$ and $Y$ are the observed responses in maps 1 and 2, respectively, at each electrode, and $X_{M}$ and $Y_{M}$ are the mean responses for each map. The magnitude of $r$ describes the strength of the association between the two sets of responses and the sign of $r$ describes the direction of this association. For each electrode, $X$ and $Y$ responses will be positively correlated when they deviate from the mean in the same direction, while they will be negatively correlated when they deviate from the mean in opposite directions.

For certain portions of our data analysis, statistically significant peak responses were extracted from the raw data. This was determined in each map by first locating each peak and trough in the raw data. Peaks were located at an electrode $i$ where the evoked response was larger than both adjacent responses at electrode positions $i-1$ and $i+1$. Similarly, troughs were located at an electrode $i$ where the evoked response was smaller than both adjacent responses at electrode positions $i-1$ and $i$ +1 . After all peaks and troughs were located by this method, a $t$ test $(P<0.05)$ was independently applied to all peak responses and each

\footnotetext{
Figure 1. Mapping synaptic input patterns onto individual cells. $A$, Arrangement of recording and stimulation electrodes in slice preparation. $B$, Evoked EPSCs for three different electrodes from a single slice experiment. Each trace is the average of four trials. The cell was located approximately $800 \mu \mathrm{m}$ from the stimulation array. Arrows indicate the stimulus artifact. $C$, Amplitude of synaptic responses evoked by each of the 24 stimulation electrodes. Each bar is the average of four trials and error bars show the standard deviation. The locations of the evoked synaptic responses shown in $B$ are labeled and marked by arrows. $D$, The data in $C$ were spatially averaged to emphasize large-scale structure in the synaptic input maps (see Materials and Methods), and was overlaid upon the raw data map. $E$, A histogram of the mean difference between evoked and summed responses as a function of electrode spacing ( $n=444$ pairs). Adjacent electrodes were $50 \mu \mathrm{m}$ apart while electrodes located $200 \mu \mathrm{m}$ apart were separated by three intervening electrodes. These calculations were carried out as part of the occlusion test to determine the independence of fiber bundles stimulated by adjacent electrodes (see Materials and Methods). The graph demonstrates that the differences between summed and evoked responses were statistically negligible across the full range of tested electrode spacings.
} 


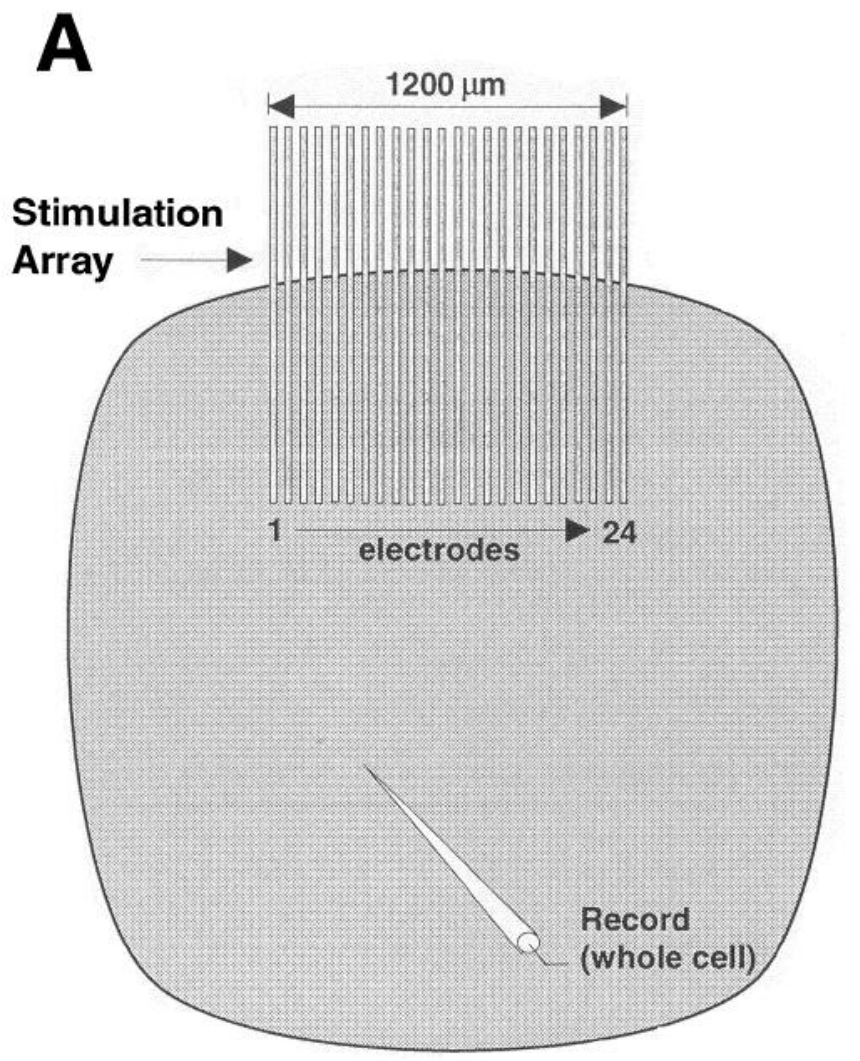

B
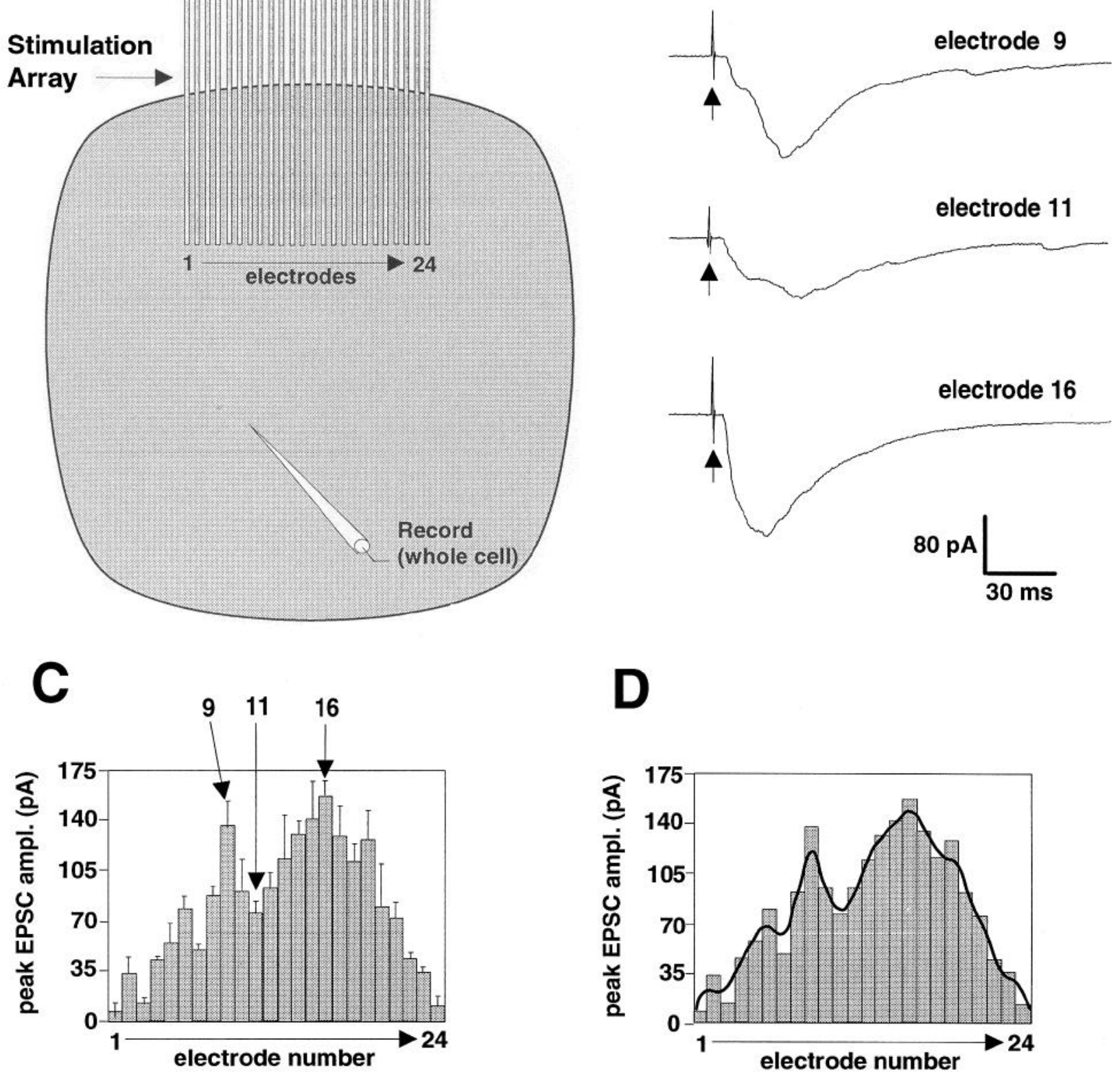

D
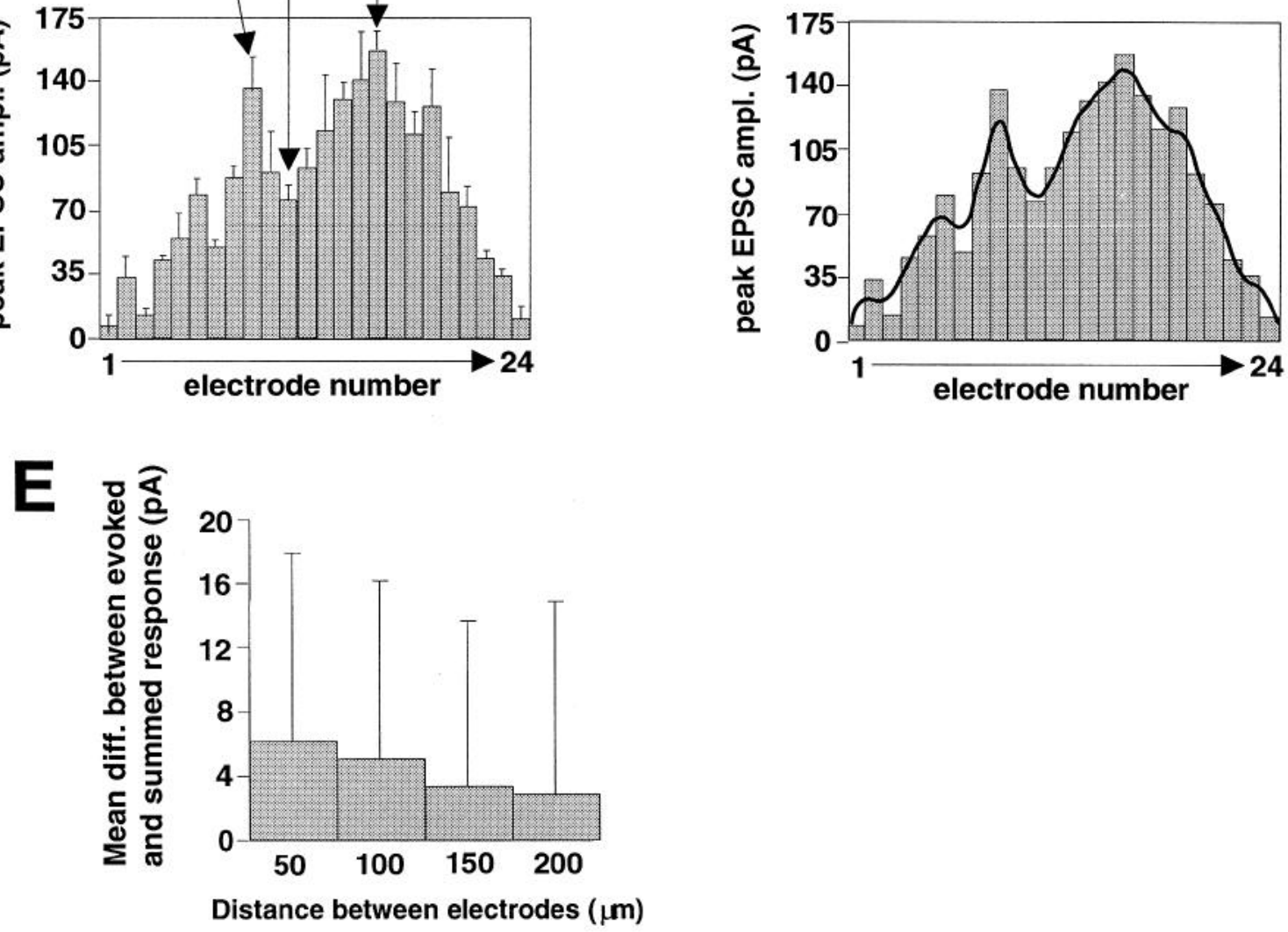
of the two closest trough responses on either side of the peak. Peaks that were statistically different than both troughs were tagged as significant. In the case of a peak with only one trough (e.g., for a peak near the edge of a map), the response at the closest edge of the map was used as a second trough as long as this response was smaller than the peak.

Optical imaging of intrinsic signals. Four ferrets (P43-P74) were anesthetized with a mixture of ketamine hydrochloride $(40 \mathrm{mg} / \mathrm{kg}, \mathrm{i} . \mathrm{m}$.) and xylazine hydrochloride ( $2 \mathrm{mg} / \mathrm{Kg}$, i.m.). During recording, animals were maintained at an anesthetized level with sodium pentothal (10-20 mg/ $\mathrm{kg}$, i.v.). They were paralyzed to prevent eye movements with pancuronium bromide (2-4 mg/ $\mathrm{kg}$, i.p.) and artificially respirated with a mixture of $70 \% \mathrm{~N}_{2} \mathrm{O}, 30 \% \mathrm{O}_{2}$ supplemented with $0.5-1.5 \%$ halothane. Heart rate and temperature of the animal were monitored throughout the duration of the experiment. The eyes were covered with a thin film of mineral oil to prevent drying. After removing the skull and dura above an area of visual cortex that included portions of areas 17 and 18 (Law and Stryker, 1988), either (1) a stainless steel chamber was cemented onto the skull, filled with mineral oil, and sealed with a glass coverglass, or (2) a glass coverglass was maneuvered into place with a micromanipulator and gently pressed against the exposed cortical surface to prevent movements due to heartbeat and respiration.

Images were acquired using an enhanced video acquisition system (Optical Imaging Inc.). The acquisition protocol was similar to previously described methods used in the cat (Bonhoeffer and Grinvald, 1993). Ferrets were monocularly stimulated through the contralateral eye with moving high-contrast square wave gratings presented on a video monitor placed $30-40 \mathrm{~cm}$ from the animal. The gratings consisted of $0.98^{\circ}$ wide bars at a spacing of $5.8^{\circ}$ and drifted at $13 \%$ sec. Gratings were drifted successively back and forth in opposite directions during each image acquisition trial, which lasted 3 sec.

Computer simulation of synaptic inputs from orientation maps. The organization of iso-orientation domains in a 74-d-old ferret is shown in Figure $7 \mathrm{~A}$. This image represents a map of orientation preferences across a region of visual cortex. To construct this map, responses were first obtained for gratings presented at eight different orientations between 0 and $158^{\circ}$. Next, differential orientation images were obtained by subtracting responses from orthogonal orientation maps (Blasdel, 1992). Intensity variations over intervals larger than $1.2 \mathrm{~mm}$ were removed by smoothing the image with an appropriate-sized convolution kernel (all pixels uniformly weighted), and subtracting the result from the original. These images were normalized by dividing the deviation from mean at each pixel by the average absolute value deviation over the entire image. The resulting eight differential images were summed vectorially to produce the orientation preference map shown in Figure $7 A$, according to previously described methods (Bonhoeffer and Grinvald, 1993). For each pixel, the orientation preference was coded according to the colors shown to the right of the image.

Synaptic input maps were calculated by placing a simulated "electrode array" onto a random location of the orientation tuning map (see Fig. 7). In addition, the array could also be positioned at a specific coordinate location in the orientation map. The array is represented by the white line seen in Figure $7 B$, and stimulation sites were all image points located under this line. Next a "recording" site was selected representing the location of a cell receiving synaptic input from all the stimulation sites. The length of the simulated stimulation array and the distance between the recording site and array were equal to those used in our experiments. As in our experiments, when recording from multiple cells in one slice, multiple simulated recording sites were chosen to be roughly the same distance from the stimulation array. Typically, input maps for 15 cells were calculated for each stimulation array location. The strength of the synaptic connection between each stimulation and recording site was varied according to the following rule: sites with the same orientation tuning were most strongly connected with a synaptic weight of 1.0 , which decreased linearly to 0.0 for sites having orthogonal orientation preferences.

\section{Results}

Our results are divided into four sections. In the first section we show that neonatal neurons in layer $2 / 3$ of ferret striate cortex receive clustered synaptic inputs via intrinsic horizontal connections. The second section demonstrates that cells in close proximity to one another receive similar patterns of synaptic inputs, and that these patterns shift in a consistent manner as the recording site moves across the cortical surface, becoming more dissimilar between cells located farther apart. In the third and fourth sections we compare experimental patterns of responses to those predicted from computer simulations, and find that most cells $(81 \%)$ receive patterns of clustered synaptic inputs predicted by the adult pattern of linked iso-orientation columns, while the remaining cells receive nonclustered inputs as predicted by a pattern of diffuse horizontal connections in young cortex. In our experiments, we observed no significant difference in the patterns of synaptic input or their modification between slices obtained from P28-P34 animals.

\section{Functional horizontal connections are clustered}

The multielectrode array (see Materials and Methods) allowed mapping of horizontal synaptic inputs onto single cells from a strip of cortex $1200 \mu \mathrm{m}$ long (Fig. $1 A$ ). In the typical slice recording and stimulation arrangement, the recording electrode was placed $500-800 \mu \mathrm{m}$ from the stimulation array. Stimulation at each of the 24 sites evoked short-latency (3-8 $\mathrm{msec}$ ) inward postsynaptic currents with peak amplitudes ranging between 10 and $250 \mathrm{pA}$, with durations of approximately $100 \mathrm{msec}$ at all locations (Fig. $1 B$ ). For each cell, an input map was constructed by plotting the amplitude of synaptic responses evoked by each of the 24 stimulation electrodes (Fig. 1C). Although cells received input from every stimulated site, the strength of the input from the different locations varied considerably. Input maps revealed that most cells received clustered synaptic inputs consisting of multiple peaks separated by regions of weaker evoked responses (Fig. 1D). Of 83 cells recorded from 15 slices, $67(81 \%)$ had maps with clustered inputs. The remaining cells had maps in which the largest synaptic inputs were elicited primarily from a single region.

In Figures $2 A, 3 A$, and $4 A$, the statistically significant peaks (see Materials and Methods) are marked by arrowheads. Most cells had maps consisting of multiple peak responses with varying distances between peaks. A large proportion ( $\sim 50 \%)$ of peaks were separated by either about $440 \mu \mathrm{m}$ or $240 \mu \mathrm{m}$ (Fig. 5). Figure 5 also shows that interpeak distances greater than about 680 $\mu \mathrm{m}$ were not common. The remaining 16 cells $(19 \%)$ had maps consisting of a primary single peak response (Fig. 2, cell 3; Fig. 4 , cells 2,5 ). The width of peak responses also varied among cells. Some were punctate, spanning only a very small region of cortex (Fig. 2, cell 1), while others were more broad and sloping, covering a much wider cortical region (Fig. 2, cell 4; Fig. 4, cell 2). Peak responses cannot be explained solely by proximity of these stimulated cortical regions to the recording site as peaks were not necessarily located at the closest electrode. For instance, peak responses were elicited from cortical areas located a farther distance from the recording site than more proximal cortical areas that elicited much weaker responses (Fig. 2, cells 1, 7; Fig. 3, cells 1-5).

Though a variety of synaptic input patterns were observed, synaptic responses always changed smoothly and continuously across the stimulated cortical region. The smooth shifting of responses between adjacent stimulated sites suggests that these patterns of synaptic input reflect an underlying functional organization in the system of horizontal connections.

\section{Patterns of horizontal connections are locally correlated}

To further determine the functional organization of horizontal connections, we examined the relationships between synaptic input maps for five to nine cells recorded in each single slice ( $n$ 

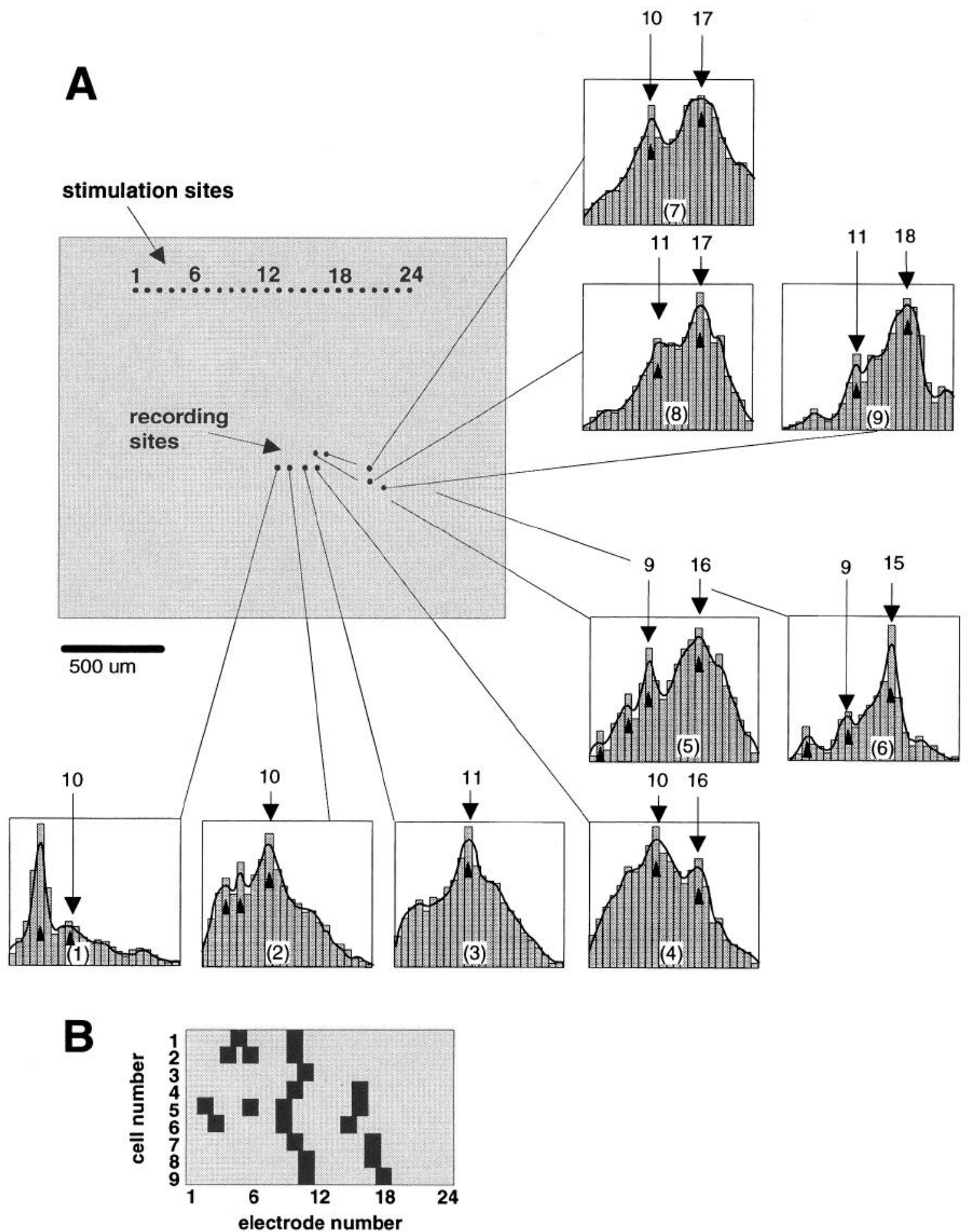

Figure 2. Nearby cells have similar patterns of synaptic inputs: results from an experiment in which input maps were acquired for nine cells in a single slice. $A$, Normalized input maps from cells located at the indicated positions within the slice. Statistically significant peaks are marked by arrowheads (see Materials and Methods). All but one cell received the largest-amplitude inputs from cortical sites stimulated by either electrodes 9-11 or 15-18. Spatially adjacent cells had similar input maps, and as the recording position shifted from left to right across the slice, the largestamplitude inputs shifted from electrodes 9-11 to 15-18. For example, cells 2, 3, and 4 had the largest input located at electrode 11, while for cells 8 and 9 the largest input was at electrodes 17-18. Cells 5 and 7, which were located at intermediate positions between cell groups $2,3,4$ and 8,9 had inputs having equivalent amplitudes located at both electrode sites. $B$, Statistically significant peaks are marked by black squares for each cell. All cell maps had a peak at electrodes 9-11 regardless of their recording position. Cells 4-9 had an additional peak at electrodes 15-18. Other cells had additional peaks at electrodes 4 and/or 6 . 


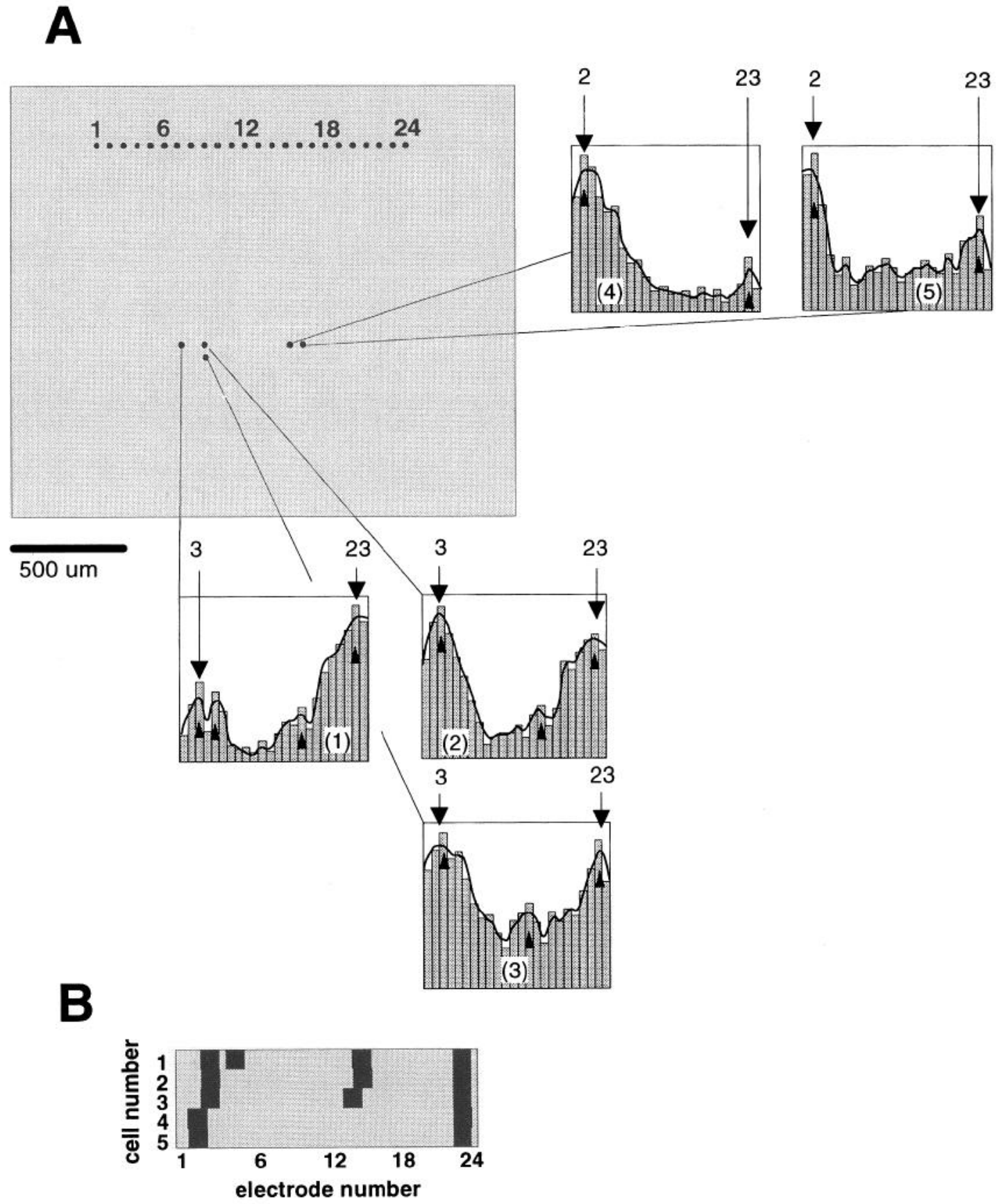

Figure 3. Shifts in response magnitudes do not correlate with proximity of the recording electrode to stimulated cortical sites. Input maps were acquired for five cells in a single slice. $A$, Same conventions as in Figure 2 . All cell maps in this experiment had peaks located at either end of the stimulation array (electrodes $2-3$ or 23 ) while receiving minimal synaptic inputs from cortical regions stimulated by the middle portions of the array. Cell 1 had the largest response from electrode 23, the most distant stimulation site, while cells 4 and 5 had largest responses from electrode 2 , also the most distant stimulation site. Cell 1 had a dominant peak response at electrode 23 and a weaker peak response at electrode 3 . As the recording position shifted to the right, cells 2 and 3 developed equal-sized peak responses at both electrodes 3 and 23 . Farther to the right, cells 4 and 5 both had dominant peak responses at electrode 2 . Cells 4 and 5 also had an additional weaker peak response at electrode 23 . Thus, the location of the peak synaptic response shifted discretely from electrode positions 23 to 2 as the recording site moved from left to right. Cells 2 and 3 , located at intermediate recording locations, had peak responses at both these electrode positions. $B$, Statistically significant peaks are marked by black squares for each cell. All cells had peak responses at electrodes 2-3 and 23. Spatially adjacent cells 1-3 also had a smaller peak response at electrodes 13-14.

$=15$ slices). Within each slice, neighboring cells received similar patterns of horizontal connections. As the distances between cells increased, however, these patterns became increasingly dissimilar (Figs. 2-4). This observation was assessed quantitatively by examining the similarity between maps of cells located at different distances (Fig. 6A). This was accomplished by calculating the degree of correlation between smoothed maps of all cell pairs within each slice ( $n=216$ cell pairs) (see Materials and Methods). For a value of $r=1$, maps are identical; this value decreases as maps become less similar. When cells were 


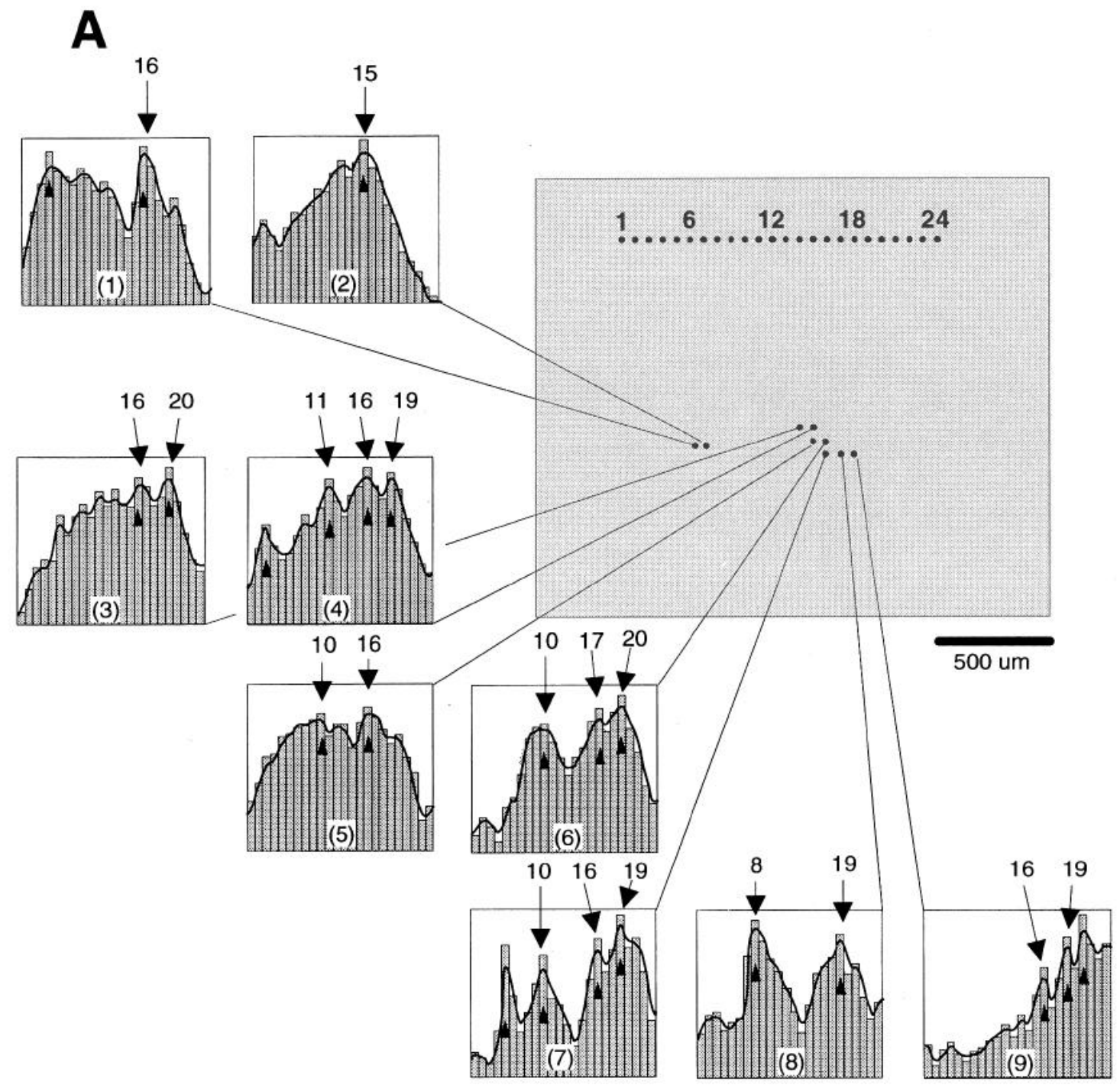

B

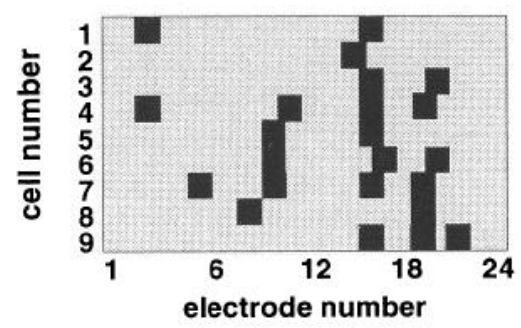

Figure 4. Patterns of synaptic response shift with changes in recording position. Input maps were acquired for nine cells in a single slice. A, Same conventions as in Figure 2. Groups of nearby cells tended to have peak responses located at the same electrode positions. Cells 1 and 2 formed one group having a dominant peak at electrodes 15-16. Cells 3 and 4 formed a second group also having a response peak at electrode 16 but with a new response peak arising at electrode 20 . Cells 6,7 , and 8 formed a third group, all having a very weakly responding area at electrodes $13-14$, which was not seen in other cells. These three cells also had large peak responses at electrodes $8-10$ and 20 . B, Statistically significant peaks are marked by black squares for each cell. Many cells had peaks located at electrodes 19 and/or 16 . Additionally, five cells (cells $4-8$ ) had peaks located at electrodes 8-11.

located close together $(<100 \mu \mathrm{m})$, synaptic input maps were highly correlated $(r=0.7)$. This correlation gradually declined to zero at about $450 \mu \mathrm{m}$. Though the overall patterns of synaptic inputs were quite different for cells separated by distances of greater than about $300 \mu \mathrm{m}$, peaks and troughs located at specific stimulated cortical locations could still be identified. For example, all cell maps in Figure 2 had a peak located within an area of cortex stimulated by electrodes $9-11$. The amplitude of this peak varied greatly for different cells: in some cell maps this peak was the largest input, while in other maps it was quite 


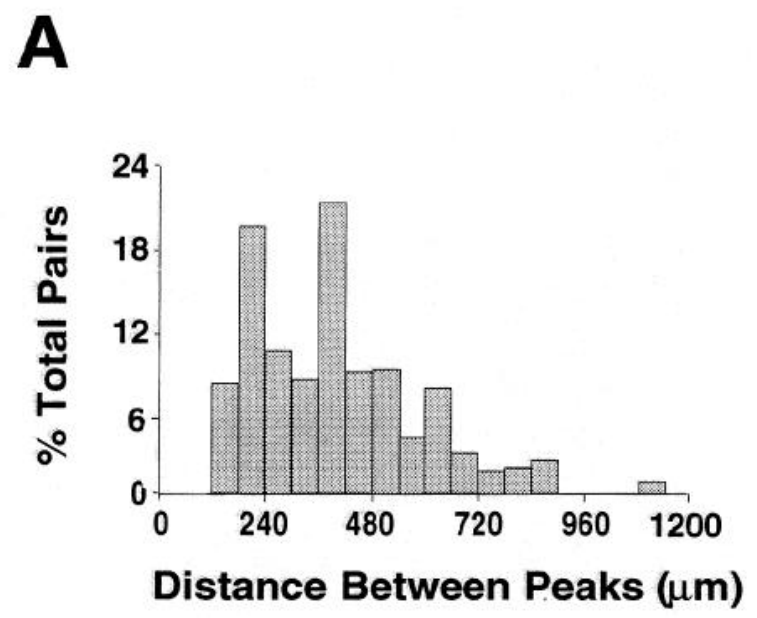

Actual Synaptic Inputs

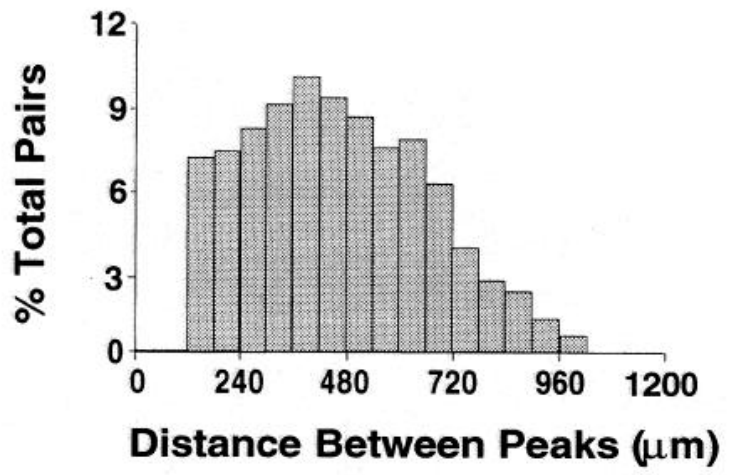

Simulated Synaptic Inputs

Figure 5. Histograms of interpeak distances for experimentally recorded and simulated linked iso-orientation maps ( $60 \mu \mathrm{m}$ bins). $A$, Experimentally recorded maps ( $n=83$ cells). A majority of peaks were separated by about $440 \mu \mathrm{m}$ or $240 \mu \mathrm{m}$. One peak was separated by $>900 \mu \mathrm{m}$. $B$, Simulated maps $(n=7500)$. Data was collected from 500 trials. For each trial, the stimulation array was placed in a different random location and maps were calculated for 15 different recording positions. All recording positions were $900 \mu \mathrm{m}$ distance from the stimulation array. Interpeak distances of about $440 \mu \mathrm{m}$ are most common. The number of instances of interpeak distances greater than $440 \mu \mathrm{m}$ gradually falls off to zero at about $960 \mu \mathrm{m}$.

small. A similar example is seen in Figure 3, where all cell maps had a peak located at electrodes $2-3$ and 23 . Figures $2 B, 3 B$, and $4 B$ show the location of all statistically significant peaks for each cell within a slice. These graphs demonstrate that many cells within each slice had peak responses evoked by stimulation of the same cortical locations.

These distinct patterns of synaptic input shifted in a consistent manner as the recording position moved across the slice. Two slices are shown in which the location of the largest synaptic input shifted discretely from one stimulated cortical location to another as the recording position was moved (Figs. 2, 3). In these slices, cells located at intermediate recording positions received large synaptic inputs from both these cortical locations. Therefore, these shifting patterns of horizontal connections further demonstrate an underlying organization to the synaptic connectivity linking different cortical sites.

\section{A model of linked iso-orientation columns reproduces the observed pattern of clustered horizontal connections}

We have demonstrated that cells receive clustered horizontal inputs from surrounding cortical locations and that synaptic responses smoothly vary from one stimulated site to the next. Furthermore, patterns of input are similar for neighboring cells and shift in a consistent manner as the recording position moves. These characteristics resemble those that might be expected from the most prominent system of horizontal connections, those related to the pattern of orientation columns. For example, orientation tuning has been demonstrated to vary smoothly across the cortical surface in a system of patchy two-dimensional domains (Fig. 7A). Importantly, anatomical studies have shown that clustered horizontal connections preferentially link together cortical columns sharing similar orientation preference (Gilbert and Wiesel, 1989).

To test specifically whether the patterns of clustered connections we experimentally observed reflect this system of linked iso-orientation columns, we attempted to reproduce these patterns using computer simulation methods. Computer-simulated "experiments," incorporating the same recording and stimulation paradigm used in our actual experiments, were performed on two-dimensional orientation tuning maps derived from optical imaging of activity-dependent intrinsic signals in ferret visual cortex (Fig. 7). We began by applying a simple connectivity rule, based upon the anatomical findings of linked isoorientation columns, to the two-dimensional orientation tuning maps. Cortical sites with the same orientation tuning were strongly connected, with the connection strength decreasing between sites with progressively more orthogonal orientation tuning preferences (see Materials and Methods). We found that simulated synaptic input maps reproduced many features of our experimental maps (Fig. 8).

The organization of iso-orientation domains in the ferret is strikingly similar to the cat (Bonhoeffer and Grinvald, 1993). "Orientation centers," around which orientation preference is organized in a pinwheel manner, are observed together with large cortical regions across which orientation preference smoothly shifts. Except for discontinuities at the pinwheel centers, orientation preference in ferret visual cortex, for the most part, varies smoothly across the cortical surface (Fig. $7 A$ ). Such smooth variations gave rise to simulated synaptic input maps displaying smooth shifts in synaptic connectivity (Figs. $7 \mathrm{C}, 8$ ), similar to our experimental maps (Figs. 2-4, 8). The simulated maps also demonstrated the presence of clustered horizontal connections, similar to the experimental maps, consisting of multiple peaks separated by areas of weaker response (Fig. 8). A histogram of interpeak distances for the simulated maps shows a maximum at $440 \mu \mathrm{m}$ (Fig. $5 \mathrm{~A}$ ), which is equal to the $440 \mu \mathrm{m}$ interpeak distance that was most commonly observed in the experimental maps (Fig. $5 B$ ).

Neighboring cells that have similar orientation preferences would be expected to receive similar patterns of synaptic inputs from surrounding cortical locations. This was demonstrated by showing that the correlation between simulated input maps was very high for neighboring sites and fell off with increasing distance (Fig. 6B). This feature was also observed for our experi- 

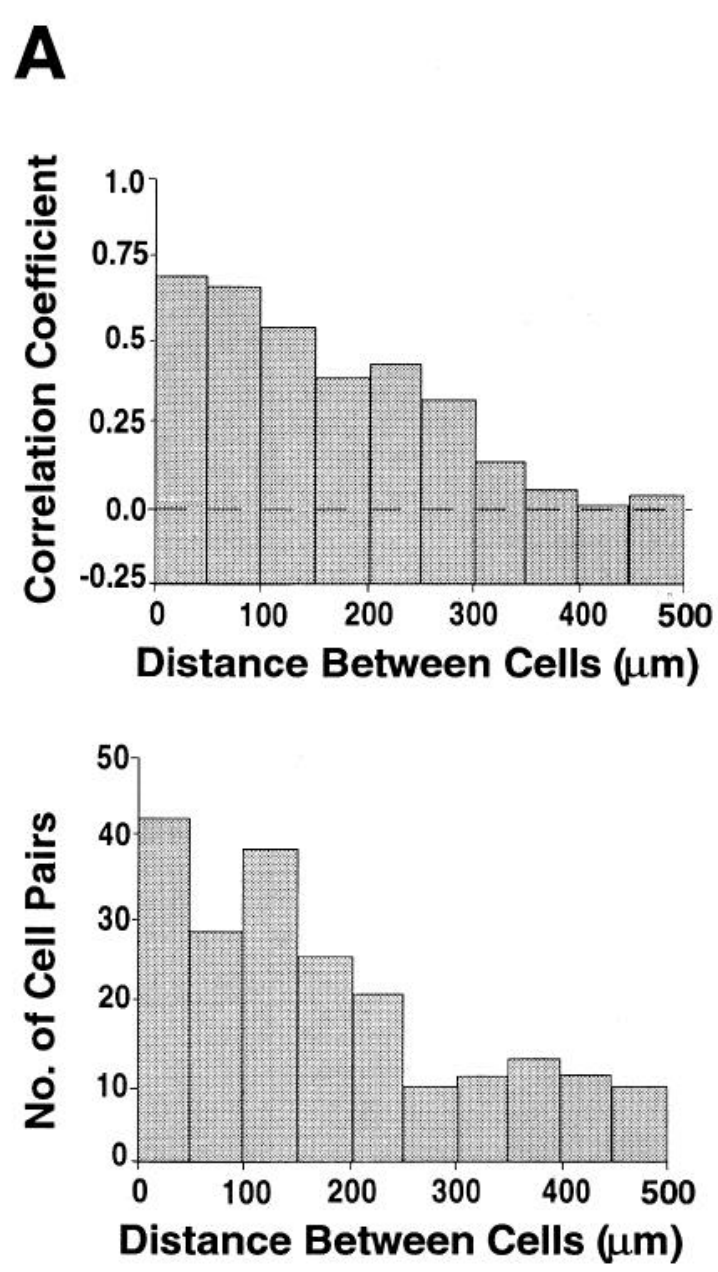

B

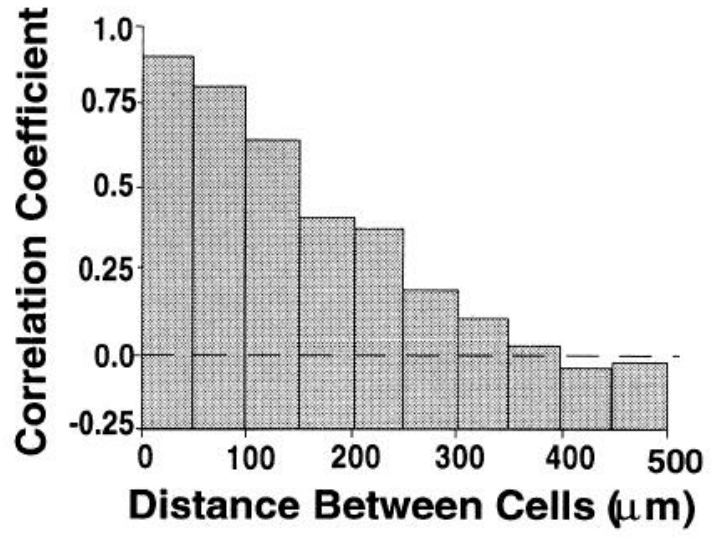

\section{Simulated Synaptic Inputs}

\section{Actual Synaptic Inputs}

Figure 6. Correlation coefficients between maps of experimentally recorded and simulated linked iso-orientation cells as a function of distance between recording sites. A, Experimentally recorded maps. The top histogram is a plot of the correlation coefficient $r$ between maps of all cell pairs in each slice versus distance between recording sites $(n=216$ pairs). Each bar shows the average correlation coefficient plotted into $50 \mu \mathrm{m}$ bins. A correlation coefficient of 1.0 means that maps are identical. Input maps are highly correlated between cells located close together; this correlation declines to zero at $450 \mu \mathrm{m}$. The lower histogram shows the number of cell pairs in each histogram bin. $B$, Simulated maps. Correlation coefficients were calculated for all cell pairs in each stimulation/recording trial ( $n=84,000$ pairs). For each trial, the stimulation array was placed in a different random location and maps were calculated for 15 different recording positions. All recording positions were $900 \mu \mathrm{m}$ distance from the stimulation array. As in experimental maps, maps for simulated cells located close together are highly correlated and this correlation falls off gradually to zero at $450 \mu \mathrm{m}$.

mental maps (Fig. 6A). Comparison of Figure 6, $A$ and $B$, shows that both experimental and simulated input maps had a correlation coefficient of zero at the same distance of $450 \mu \mathrm{m}$ separation between recording sites. This value is close to the 470 $\mu \mathrm{m}$ average distance between sites having orthogonal orientation preferences, calculated from the orientation tuning map shown in Figure 7. This is not surprising, since cortical sites having orthogonal orientation preferences would be linked to different surrounding cortical locations and would therefore have very dissimilar input maps.

Interestingly, the simulated maps reproduced the consistent manner in which experimental synaptic input patterns shifted with changes in recording position (Fig. 8). The simulations showed that changes in orientation tuning at different recording positions gave rise to corresponding shifts in the pattern of synaptic inputs. For instance, in Figure $8 B$ orientation prefer- ence smoothly shifts from purple $\left(85^{\circ}\right)$ to orange $\left(158^{\circ}\right)$ as the recording position moves from position 1 to 3 . Since cortical sites with the same orientation tuning (i.e., same color) are preferentially connected, location "b" (purple) on the stimulation array generates the largest response at recording site 1 (purple). Likewise, location location "a" (orange) generates the largest response at recording site 3 (orange). However, both "a" and "b" generate equivalent responses at recording site 2 , which has an intermediate orientation tuning between recording sites 1 and 3. A similar shift in synaptic responses was observed for the series of experimental maps shown in Figure 8A, suggesting that there was a similar shift in synaptic connectivity as observed in the simulated maps. In a second example, an experimentally observed single peak split into two separate peaks as the recording position moved across the slice (Fig. $8 \mathrm{C}$ ). This behavior was reproduced by the simulation when the "stimulation array" 


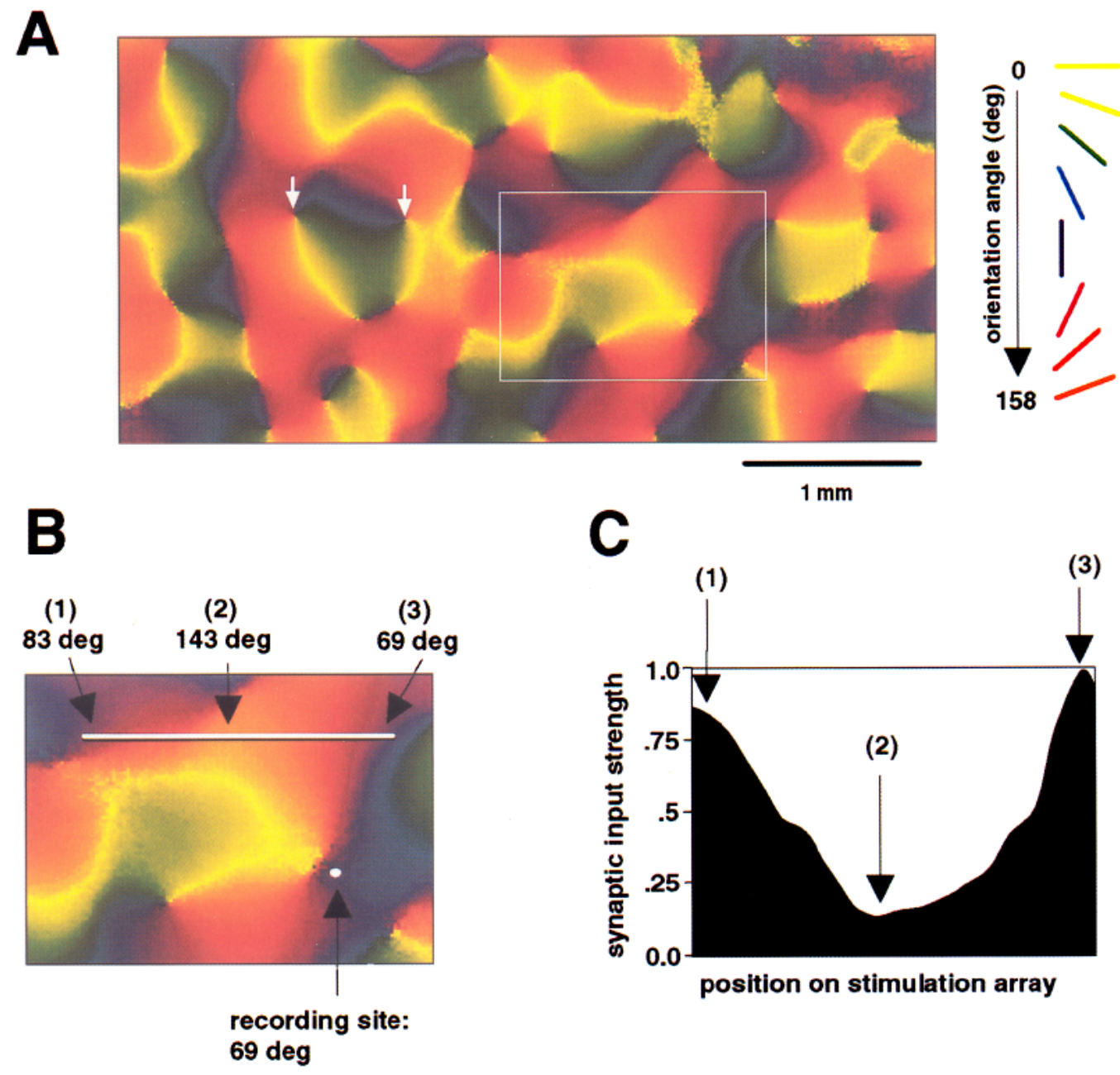

Figure 7. Method for calculating simulated synaptic input maps. A, A map showing the organization of iso- orientation domains in ferret visual cortex was derived from optical imaging of activity-dependent intrinsic signals (see Materials and Methods). The orientation angle of best response for each point in the image was coded according to the colors shown to the right. Arrows point to 2 "orientation centers" around which orientation preference is organized in a pinwheel manner as previously demonstrated in cat visual cortex. $B$, The area bounded by the white square in $A$ has been expanded. The stimulation array is represented by the white line and a recording position is marked. The length of the stimulation array is $1200 \mu \mathrm{m}$. $C$, The distribution of simulated horizontal synaptic connections between the stimulation sites and recording position in $B$. Three corresponding positions along the stimulation array have been labeled in this panel and in $B$. Position 1 had an orientation preference of $83^{\circ}$ while the recording site had a preference of $69^{\circ}$, producing an orientation tuning difference between the two sites of $14^{\circ}$. Since the strength of the synaptic connection between sites varied from 1.0 to 0.0 as the orientation tuning difference varied from 0 to $90^{\circ}$, the synaptic weight between stimulation position 1 and the recording site was $(90-14) /(90)=0.84$. This has been graphed and labeled $(1)$. The orientation tuning difference between stimulation site 2 and the recording site was $74^{\circ}$. Therefore, the synaptic weight between these two sites was $(90-74) /(90)=0.17$ as graphed. The same process was used to calculate the synaptic weights between stimulation position 3 and the recording site, as well as the remaining stimulation sites along the array. For this stimulation and recording arrangement, a synaptic input map was generated, having two large peaks at either end of the stimulation array separated by a region of weaker synaptic connectivity.

traversed the rounded corner of an orientation domain (Fig. $8 D)$.

\section{A radial falloff model reproduces the observed pattern of nonclustered horizontal connections}

A number of experimentally recorded cells had synaptic input distributions that deviated significantly from those predicted by the model of linked iso- orientation columns. Input maps from these cells support an alternative model in which horizontal connections are uniformly distributed throughout the cortex but decline radially in strength from a source cortical site. A very specific pattern of synaptic inputs is predicted by this model (Fig. 9). Since the strength of synaptic input onto a cell falls off with increasing distance, an input map is produced having a single peak located at a position along the stimulation array nearest to the cell. Synaptic input falls off gradually to either side of this peak along the stimulation array. The falloff was modeled by a sine function having maximum amplitude at 0 $\mu \mathrm{m}$ and zero amplitude at $600 \mu \mathrm{m}$. For instance, in Figure $9 A$, a cell located at recording site 1 would have a peak synaptic input at stimulation site " $b$," which lies directly across from the cell on the stimulation array. Shifting the recording site relative to the stimulation array causes a corresponding shift of this peak, which is seen in the input maps of cells 2 and 3 (Fig. 9B). Figure $9 C$ shows experimental synaptic input maps for three cells recorded from two different slices. These cells were recorded at 
A

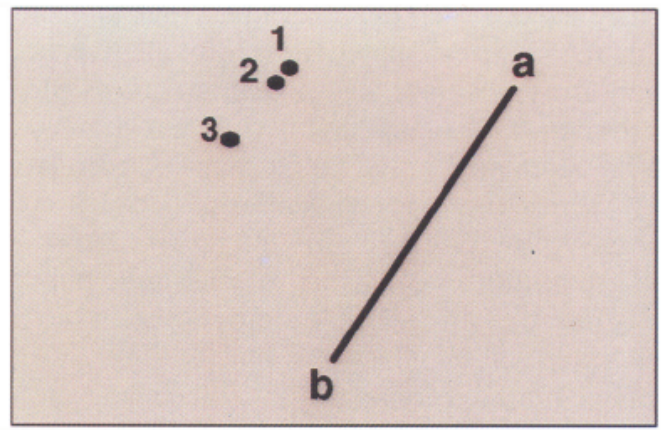

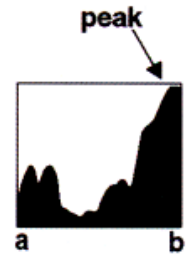

1

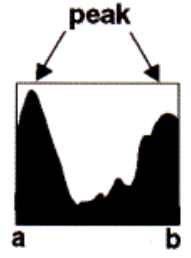

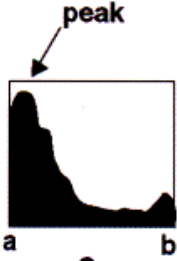

3

\section{Actual Synaptic Inputs}
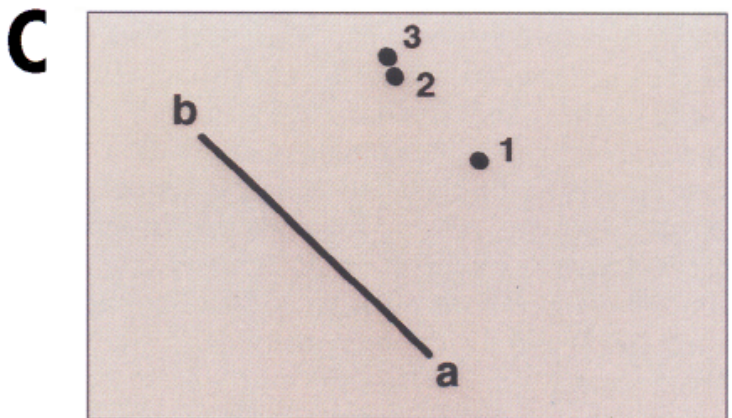

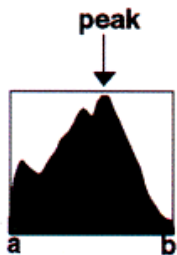

1

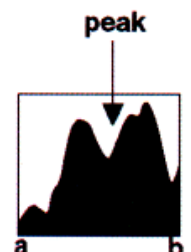

2

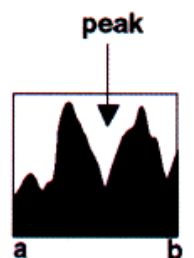

3

D

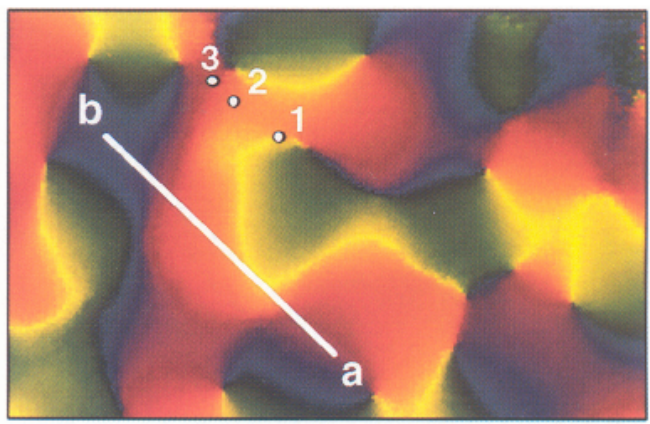

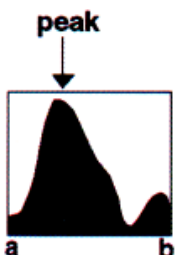

1

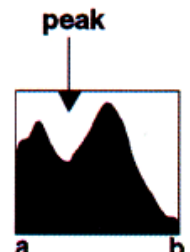

2

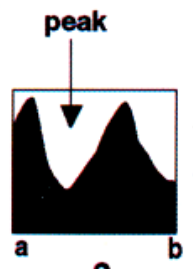

3

\section{Actual Synaptic Inputs}

\section{Simulated Synaptic Inputs}

Figure 8. Experimentally recorded maps are compared to simulated maps derived from the model of linked iso-orientation columns (see Fig. 7 for the method of calculating simulated maps). In each panel, locations $a$ and $b$ on the stimulation array correspond to points $a$ and $b$ on the synaptic input maps. In all panels, the stimulation array is $1200 \mu \mathrm{m}$ long. $A$ and $C$ show experimentally obtained synaptic input distributions from two different slices, while $B$ and $D$ show that simulated synaptic input maps reproduced many aspects of the experimental data. Both experimental and simulated input maps showed the existence of strongly clustered horizontal connections consisting of peak responses separated by regions of weaker synaptic input. Synaptic responses smoothly decreased from these peaks as the cortical stimulation site shifted along the array. $A$ and $B$, Peak responses are observed at both ends of the stimulation array. The location of the peak synaptic response shifted from electrode position $b$ to $a$ as the recording location moved from cell 1 to 3 . Interestingly, cell 2, which lies at an intermediate location between cells 1 and 3 , had two peak responses that were at the same locations as the individual peak responses of cells 1 and 3. $C$ and $D$, Cell 1 had an input map consisting of a single large peak. As the recording site shifted to the left at cell positions 2 and 3, this single peak split into two separate peaks located to either side of the original 

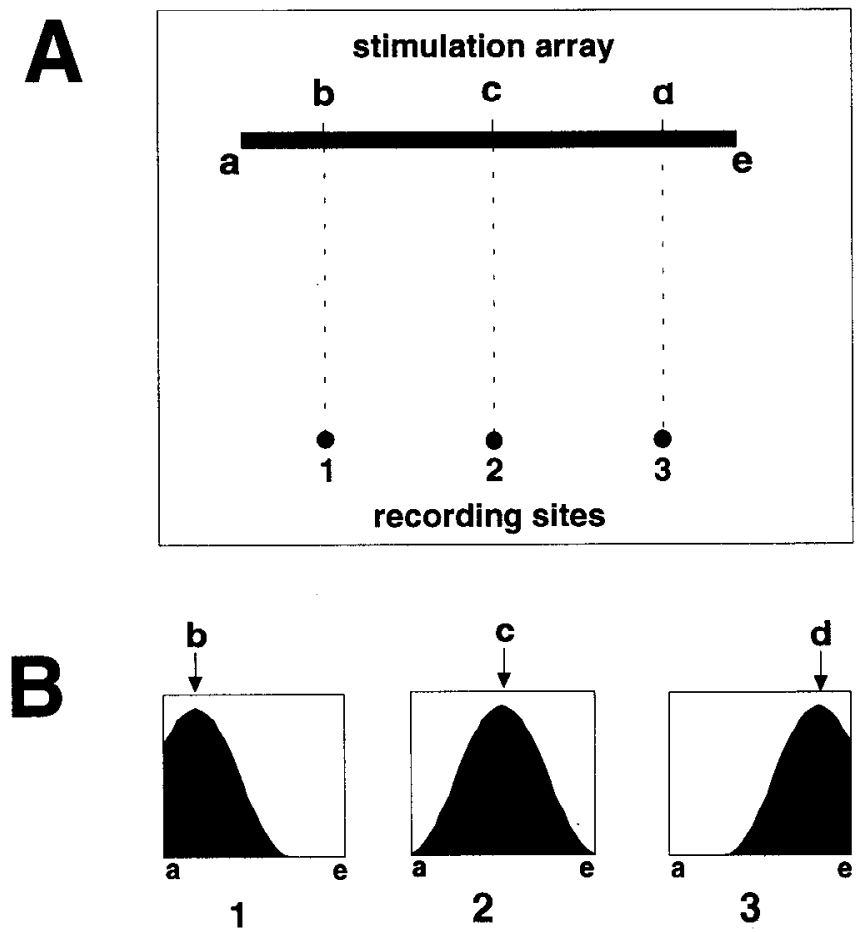

Simulated Synaptic Inputs

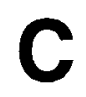

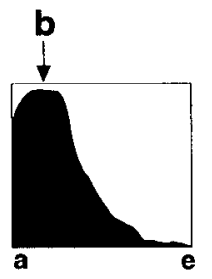

1

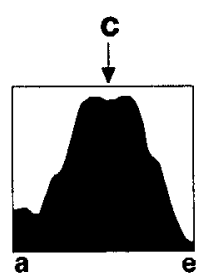

2

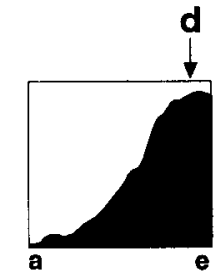

3

\section{Actual Synaptic Inputs}

Figure 9. Simulated and experimental maps predicted by a model where the strength of horizontal synaptic connections falls off radially from a source cortical site. $A$, Recording and stimulation arrangement for demonstrating the radial falloff model. Recording positions 1,2 , and 3 are located closest to respective sites $b, c$, and $d$ along the stimulation array. The same recording and stimulation sites are used for both $B$ and $C$ as follows. $B$, Simulated maps. Cells 1,2 , and 3 have a single peak response located at stimulation sites $b, c$, and $d$, respectively. The peak is always at a location along the stimulation array closest to, and directly opposite, the recording site. Shifting the recording position causes the location of the peak response to correspondingly shift along the stimulation array. $C$, Experimentally recorded maps for three cells were recorded from two different slices. Similar to the simulated maps, each cell has a single peak response that is at a location along the stimulation array closest to the recording site.

positions 1, 2, and 3 as shown in Figure $9 A$. Each cell's map has a single peak located at a position along the stimulating array closest to that cell. These experimental maps closely resemble the simulated maps shown in Figure $9 B$.

Calculations were carried out to determine the similarity of experimental input maps and simulated maps derived from the model of linked iso-orientation columns, to the radial falloff model. Figure $10 \mathrm{~A}$ shows the result of this calculation for the experimentallv acauired mans. Correlation coefficients were cal- culated between each experimental input map and a simulated radial falloff map calculated from equivalent recording and stimulation array position. The histogram shows the percentage of experimental cell maps having different degrees of correlation with the simulated radial falloff maps. A correlation coefficient of 1.0 represents complete similarity between maps, while low or negative coefficients represent no similarity. There is a bimodal distribution consisting of one large population of cells having negative or negligible similarity with the model and another group having strong similarity. The similarity between simulated maps, generated by the linked iso- orientation model, and maps generated by the radial falloff model is negligible (Fig. $10 B)$.

\section{Discussion}

The pattern of clustered horizontal connections of pyramidal cells in the striate cortex is intimately associated with the organization of orientation-specific domains. These clusters are among the earliest anatomical features of cortical functional architecture. Crude clusters have been detected as early as postnatal day 8 in the cat, even prior to eye opening; by P14 crude clusters are clearly present and occupy the same position as adult clusters (Callaway and Katz, 1990). This is well prior to the emergence of ocular dominance columns and occurs at a time when orientation tuning is not yet detectable in many neurons (Braastad and Heggelund, 1985). Prcliminary cvidence in ferrets (J. Durack and L. C. Katz, unpublished observations) suggests that crudely clustered axonal collaterals are present by about P30, before eye opening. This is at least a week before most cells in ferret striate cortex are strongly tuned for orientation (Chapman and Stryker, 1993). In both cats and ferrets, the gradual increase in the number of orientation-tuned neurons and the precision of clustered connections between similarly tuned groups occurs roughly in parallel. Neuronal activity, and in particular correlated patterns of activity, are clearly critical for the emergence both of orientation tuning (Chapman and Stryker, 1993) and for the refinement of clustered connections (Callaway and Katz, 1991; Löwel and Singer, 1992). On a theoretical level, it has been suggested that the presence of tangential connections could determine the periodicity and organization of orientation columns (von der Malsburg and Cowan, 1982). $\Lambda \mathrm{n}$ obligatory requirement for an activity- and correlation-based mechanism for the emergence of clustered connections is the presence of functional connections between the relevant groups of neurons. A central issue, then, is whether the patterns of projections and developmental rearrangements observed using anatomical approaches are indicative of the presence of patterns of functional synaptic connections.

Most investigations into the development of the system of horizontal connections have relied on a variety of anatomical assays to infer the state of functional connections (Callaway and Katz, 1990, 1991; Löwel and Singer, 1992; Lubke and Albus, $1992 a, b)$. For example, in previous work using intracellular dye injections in brain slices, it was found that cells initially elaborated long, unbranched collaterals, with few secondary branches. It was assumed that these axons formed functional synapses with roughly equal probability along their length, and that an increased density of collateral branching implied an increased density of synaptic contacts (Callaway and Katz, 1990). Because the sites of actual synapses in the immature brain are often difficult to detect anatomically (the axonal swellings typical of en passant svnanses in the adult are not vet develoned). it was 

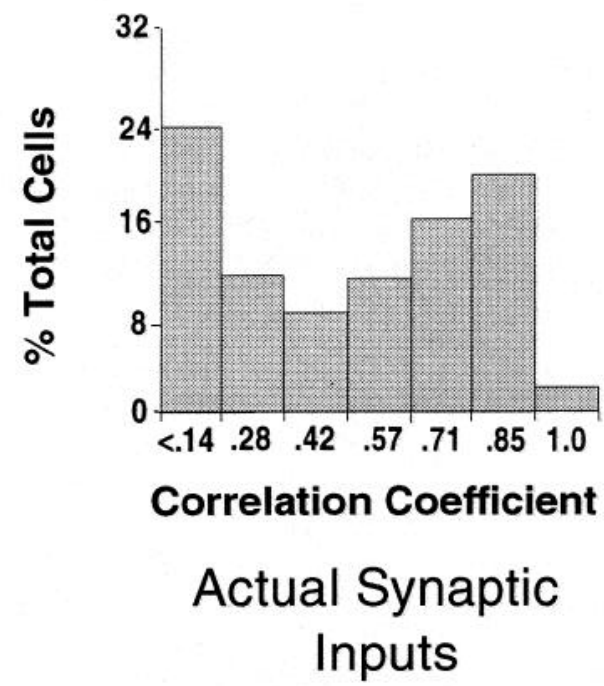
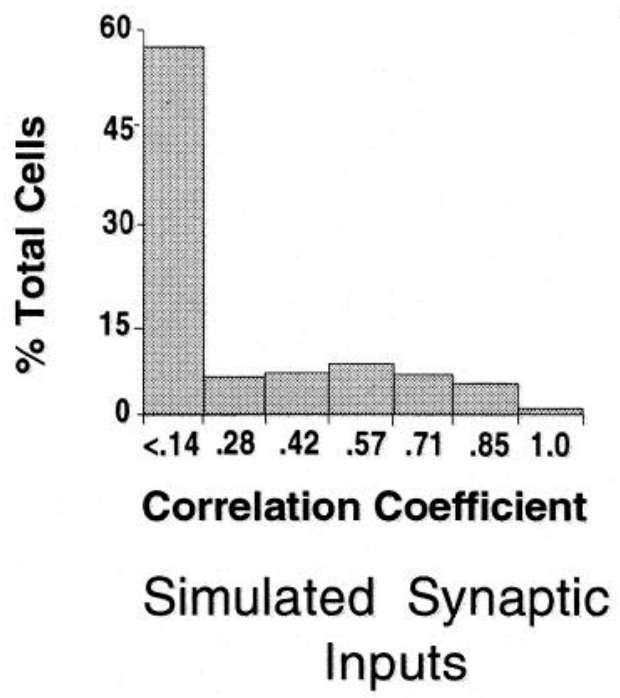

Figure 10. Histograms show the percentage of experimental and simulated linked iso-orientation maps having different degrees of correlation with maps generated by the radial falloff model. $A$, Experimental maps. There is a bimodal distribution of cells having either negligible or strong correlations with the radial falloff model. $B$, Simulated maps. Almost all linked iso-orientation cells have negligible correlations with the radial falloff model $(n=800$ cells $)$.

not clear if these early tangential axons were even forming functional connections. The results presented here, demonstrating the existence of functionally clustered horizontal connections, imply that by P30 many or all of the collaterals are forming functional connections. Our results further indicate that even prior to eye opening, and well before substantial visual experience, a considerable degree of functional organization already exists in the system of cortical horizontal connections. As the subsequent development of this system relies on neural activity, we surmise that this initial patterning is produced by spontaneous activity in the developing cortex (Goodman and Shatz, 1993).

\section{Determining patterns of functional synaptic connections in the developing cortex}

Determining the functional state of horizontal connections onto single cells is difficult in the immature visual system. Prior to and shortly after the time of eye opening, conventional in vivo electrophysiological techniques are of limited use. Because of the low and variable levels of evoked and spontaneous activity, cross-correlation methods (T'so et al., 1986; Hata et al., 1993), which reveal horizontal connections in older animals, are impractical. Other techniques that could reveal the functional state of horizontal connections (such as surround stimulation) are compromised by the optics of the eye and weak overall responses of cortical cells around the time of eye opening (Hubel and Wiesel, 1963; Braastad and Heggelund, 1985). Electron microscopic analyses can in principle determine the presence of synapses, but reconstructing large-scale patterns using this approach is exceedingly difficult. Light microscopic techniques are also of limited use: the characteristic swellings and varicosities that identify en passant synaptic boutons in adult cortical neurons are poorly differentiated in young animals. Furthermore, the presence of horizontal inputs determined by these various an- atomical or physiological techniques does not reveal the spatial pattern of these inputs with fine resolution.

Recently, in vitro techniques for assessing the functional state of connections in the developing cortex have been developed. Scanning laser photostimulation using caged glutamate (Callaway and Katz, 1993; Katz and Dalva, 1993) can provide detailed two-dimensional maps of the patterns of synaptic inputs onto single cortical neurons during the development of horizontal connections (Dalva and Katz, 1993). Despite the power of this technique, the speed with which maps can be made makes it is difficult to record patterns of more than one or two cells per slice. It is therefore difficult to determine whether the patterns of horizontal inputs onto nearby cells are similar, and therefore more likely to reflect an overall pattern of connections present in the slice. Unlike photostimulation, the multielectrode stimulation array described in this article can produce only onedimensional maps of inputs onto a given cell, and these maps may also be contaminated by activation of fibers of passage. Despite these limitations, the array makes it possible to map the input pattern rapidly onto a given cell, and to determine the patterns for numerous closely spaced neurons.

\section{Patterns of functional synaptic connections reflect patterns of horizontal anatomical projections}

Our results indicate that most cells receive nonhomogeneous patterns of synaptic inputs at this early developmental stage. What do these patterns of inputs represent? Based on several lines of eyidence, including the spacing between peaks of activity, the correlated patterns of nearby cells, and the correspondence between experimental and simulated input maps, we conclude that the observed patterns of synaptic connections reflect the presence of clustered horizontal inputs linking cortical columns that have, or will have, similar orientation tuning.

In our experimental maps, a majority of cells had interpeak 
distances of $440 \mu \mathrm{m}$ and $220 \mu \mathrm{m}$. This is smaller than the typical distance of 800-1000 $\mu \mathrm{m}$ between clustered horizontal connections observed anatomically in the cat cortex (Gilbert and Wiesel, 1983; Callaway and Katz, 1990). However, our recordings were done using a randomly oriented stimulation array that traversed orientation domains in an unknown fashion. In some cases we would expect the array to run parallel to an iso-orientation domain, in other cases to cut through edges or corners of such domains (see Fig. 8). The recording electrodes were also placed essentially randomly, in the sense that we had no idea if we were at the center or edge of an iso-orientation domain. In such cases the distances between peaks would deviate considerably from the millimeter or so seen with anatomical methods. That this is in fact the case was revealed by simulating the data using an orientation map derived from optical imaging experiments. In the simulations, the distances between peaks also averaged about $480 \mu \mathrm{m}$. Thus, the interpeak distances observed experimentally are almost precisely those expected from a set of inputs originating from an organized pattern of orientation domains.

The strong correlations between the patterns of inputs of nearby cells are also consistent with input patterns originating from clustered horizontal connections. For any one cell, a nonuniform pattern of inputs could result from any number of artifactual reasons, including truncation of axons, stimulation of axons of passage, or truncation of the dendrites of the recorded cell. It is much less likely that any of these explanations could account for the consistently observed degree of correlation between neighboring cells, or for the smooth shifting of patterns with increasing distance. The relatively high degree of correlation of input patterns amongst cells separated by $<500 \mu \mathrm{m}$ is also consistent with several other observations on the structure of clustercd conncctions. The clustered patches of cells labeled after a small injection of a retrograde tracer into layer $2 / 3$ occupy regions 300-500 $\mu \mathrm{m}$ in diameter (Gilbert and Wiesel, 1989; Callaway and Katz, 1990). Our results imply that cells within such patches are receiving similar patterns of inputs. This agrees very well with in vivo results using cross-correlation analysis. During the equivalent developmental stage in the cat (P7-P14, which corresponds to about P28-P34 in ferret), Hata et al. (1993) found that about $20 \%$ of cell pairs showed correlated firing patterns, mostly due to common excitatory inputs. Such correlations were seen only when electrodes were within $600 \mu \mathrm{m}$ of each other. Hata et al. (1993) also noted that the correlations, even at the youngest ages, were primarily between neurons with similar orientation sensitivitics.

Although most cells had input maps that were well fit by our model of linked iso-orientation columns, about $20 \%$ of the experimentally recorded cells had input maps that supportcd a model of uniformly distributed horizontal connections radially decreasing in strength from a source site. Such cells were typically found in slices that also contained cells with input maps predicted by the linked iso-orientation model, although in a few slices we found cells of almost exclusively one type or another. There are several possible explanations for the existence of these patterns of synaptic input onto single cells. These could be developmentally younger cells receiving horizontal connections that are still in an unclustered state (Callaway and Katz, 1991; Dalva and Katz, 1993). Since the cortex develops in a roughly inside-out manner, cells near the top of layer $2 / 3$ could be more immature than deeper-lying cells; this was certainly observed anatomically in the development of vertical connections of layer
$2 / 3$ cells in the cat (Katz, 1990). It is also possible that different cell types receive different patterns of horizontal connections; there is as yet no concensus on whether all pyramidal neurons either make or receive clustered connections. Indeed, some of the recorded neurons may not have been pyramidal cells, and it is unknown whether inhibitory neurons receive clustered inputs (although recent results suggest that at least some do; Dalva and Katz, 1994). Since we did not intracellularly fill recorded cells, we were unable to identify them morphologically. Recordings werc also made from cells at various depths within each slice, and slices were cut from the upper layers of cortex that might have included not only layer $2 / 3$ but also parts of layer 4 . Layer $2 / 3$ pyramidal cells have clustered branches in layers $2 / 3$ and 5 , not in layer 4 , and layer 4 cells themselves do not form clustered connections (Gilbert and Wiesel, 1979, 1983; Martin and Whitteridge, 1984). Finally, we cannot rule out artifactual contributions to our maps from stimulations of axons of passage and polysynaptic activation. For the majority of experiments, in which neurons located close together had correlated input maps, it is unlikely that these potential artifacts made a significant contribution; in a few cases, however, these contributions may have contaminated the precision of maps.

Despite the considerable evidence that clustered inputs are the physiological correlate of clustered tangential connections, definitive proof will require experiments in which the orientation selectivities of both the electrodes in the stimulating array and the recording electrode are known. In our simulated experiments these were known, and the results of those simulations agree well with the experimental results. In experiments currently underway, we have used optical recording in vivo to generate orientation maps of a portion of the cortex; that portion is subsequently removed and used for preparing brain slices for experiments like those described here. It should thus be possible to determine directly whether stimulation of different orientation domains gives rise to a pattern of inputs onto individual cells as predicted by a model of functionally linked iso-orientation columns.

\section{References}

Aghajanian GK, Rasmussen K (1989) Intracellular studies in the facial nucleus illustrating a simple new method for obtaining viable motoneurons in adult brain slices. Synapse 3:331-338.

Blanton MG, Lo Turco JJ, Kriegstein AR (1989) Whole cell recording from neurons in slices of reptillian and mammalian cerebral cortex. J Neurosci Methods 30:203-210.

Blasdel GG (1992) Differential imaging of ocular dominance and orientation selectivity in monkey striate cortex. J Neurosci 12:31153138.

Bonhoeffer T, Grinvald A (1993) The layout of iso-orientation domains in area 18 of cat visual cortex: optical imaging reveals a pinwheel-like organization. J Neurosci 13:4157-4180.

Braastad OB, Heggelund P (1985) Development of spatial receptivefield organization and orientation selectivity in kitlen striate cortex. J Neurophysiol 53:1158-1178.

Bradler JE, Barrionuevo G (1989) Long-term potentiation in hippocampal CA3 neurons: tetanized input regulates heterosynaptic efficacy. Synapse 4:132-142.

Callaway EM, Katz LC (1990) Emergence and refinement of clustered horizontal connections in cat striate cortex. J Neurosci 10:1134-1153. Callaway EM, Katz LC (1991) Effects of binocular deprivation on the development of clustered horizontal connections in cat striate cortex. Proc Natl Acad Sci USA 88:745-749.

Callaway EM, Katz LC (1993) Photostimulation using caged glutamate reveals functional circuitry in living brain slices. Proc Natl Acad Sci USA 90:7661-7665.

Chapman B, Stryker MP (1993) Development of orientation selectiv- 
ity in ferret visual cortex and effects of deprivation. J Neurosci 13: 5251-5262.

Dalva MB, Katz LC (1993) Scanning laser photostimulation reveals development of synaptic connections of clustered horizontal projections in ferret visual cortex. Soc Neurosci Abstr 19:240.

Dalva MB, Katz LC (1994) Scanning laser photostimulation reveals rearrangements of intrinsic synaptic connections in developing visual cortex. In press.

Gilbert CD, Wiesel TN (1979) Morphology and intracortical projections of functionally identified neurons in cat visual cortex. Nature 280:120-125.

Gilbert CD, Wiesel TN (1983) Clustered intrinsic connections in cat visual cortex. J Neurosci 3:1116-1133.

Gilbert CD, Wiesel TN (1989) Columnar specificity of intrinsic horizontal and corticocortical connections in cat visual cortex. J Neurosci 9:2432-2442.

Glantz SA (1981) Primer of biostatistics. New York: McGraw-Hill.

Goodman CS, Shatz CJ (1993) Developmental mechanisms that generate precise patterns of neuronal connectivity. Cell [Suppl] 72:7798.

Hata Y, Tsumoto T, Sato H, Hagihara K, Tamura H (1993) Development of local horizontal interactions in cat visual cortex studied by cross-correlation analysis. J Neurophysiol 69:40-56.

Hubel DH, Wiesel TN (1963) Receptive fields of cells in striate cortex of very young, visually inexperienced kittens. J Neurophysiol 26:9941002.

Katz LC (1990) Specificity in the development of vertical connections in cat striate cortex. Eur J Neurosci 3:1-9.

Katz LC, Dalva MB (1993) Elucidating the physiology of developing local circuits in visual cortex: scanning laser photostimulation in vitro. Soc Neurosci Abstr 19:240.
Law MI, Stryker MP (1988) Organization of primary visual cortex (area 17) in the ferret. J Comp Neurol 278:157-180.

Livingston MS, Hubel DH (1984) Specificity of intrinsic connections in primate primary visual cortex. $J$ Neurosci 4:2830-2835.

Löwel S, Singer W (1992) Selection of intrinsic horizontal connections in the visual cortex by correlated neuronal activity. Science 255:209212.

Lubke J, Albus K (1992a) Lack of exuberance in clustered intrinsic connections in the striate cortex of one-month-old kitten. Eur J Neurosci $4: 189-192$.

Lubke J, Albus K (1992b) Rapid rearrangement of intrinsic tangential connections in the striate cortex of normal and dark-reared kittens: lack of exuberance beyond the second postnatal week. J Comp Neurol 323:42-58.

Malach R, Amir Y, Harel M, Grinvald A (1993) Relationship between intrinsic connections and functional architecture revealed by optical imaging and in vivo tangential biocytin injections in primate striate cortex. Proc Natl Acad Sci USA 90:10469-10473.

Martin KAC, Whitteridge D (1984) Form, function and intracortical projections of spiny neurons in the striate visual cortex of the cat. $J$ Physiol (Lond) 353:463-504.

Rockland KS, Lund JS (1982) Widespread periodic intrinsic connections in the tree shrew visual cortex. Brain Res 169:19-40.

Rockland KS, Lund JS (1983) Intrinsic laminar lattice connections in primate visual cortex. J Comp Neurol 216:303-318.

T'so DY, Gilbert CD, Wiesel TN (1986) Relationships between horizontal interactions and functional architecture in cat striate cortex as revealed by cross-correlation analysis. J Neurosci 6:1160-1170.

von der Malsburg C, Cowan JD (1982) Outline of a theory for the ontogenesis of iso-orientation domains in visual cortex. Biol Cybern 45:49-56. 